\title{
The later clinical course of haematemesis
}

\author{
J. SHAFAR \\ M.D., F.R.C.P., D.P.H.
}

Physician
J. P. MIDGLEY
M.B., Ch.B.

Registrar

The Burnley Group of Hospitals

\begin{abstract}
A study was conducted on the natural history of haematemesis in patients with peptic ulcer and in those with negative radiological examination, the bleed having occurred at least 5 years previously; excluded were those who had undergone emergency or interim surgery.
\end{abstract}

Summary

No significant difference was found in the average severity of the bleed between the ulcer and non-ulcer groups, which were comparable in age-distributions.

Routine follow-up after the haemorrhage was discontinued following correction of the post-haemorrhagic anaemia; the present haematological status was found to be satisfactory in the very great majority and it appears that the acute bleed bears little if any relationship to the process of chronic blood loss.

Physical examination and liver function tests revealed no evidence of delayed hepatic damage ensuing as a consequence of transfusion.

Recurrent haemorrhages occurred in similar proportions in the ulcer and non-ulcer groups; they revealed no particular time interval or features of significant distinction from those of the initial bleed.

Persistence of dyspepsia showed no correlation with a bleeding tendency.

There is a preponderance of blood group $\mathbf{O}$ in the ulcer and non-ulcer bleeders.

The radiological presence of a peptic ulcer does not necessarily indicate that this is the source of the bleeding and it is suggested that the comparable features of the select group of ulcer cases of our series and the non-ulcer cases offer support to the view that the origin of the bleed in a fair proportion of instances is common to the two groups; the possibility of a derangement of the microvasculature as the operating mechanism is mooted.

\section{Introduction}

Analyses of haematemesis tend to centre round the immediate event; the subsequent clinical course has not received commensurate attention. The present investigation was instituted with the latter aspect in mind and has also afforded an opportunity for comparison of the later clinical behaviour of those affected with peptic ulcer and those in whom clinical and radiological examination had revealed no apparent causal pathology for the bleed.

\section{Materials and methods}

The patients had all received treatment in hospital for the original haemorrhage. A 5-year interval from this episode was the minimal permissible period for inclusion in the study which was also dependent either on the radiological demonstration of a peptic ulcer or on a negative X-ray report. Our criteria excluded conditions such as hiatus hernia and neoplasm; in the X-ray-negative cases existing disorders such as pseudoxanthoma elasticum and haemorrhagic telangiectasia, and also those who underwent emergency surgical intervention or an elective operation prior to the lapse of the 5-year period. A personal interview was conducted with each patient. Venous blood was submitted for haemoglobin determination $(14.6 \mathrm{~g}=100 \%)$ and for the assessment of liver function; the tests comprised the serum level of bilirubin, albumin, thymol turbidity, zinc sulphate turbidity and alkaline phosphatase. The routine practice following discharge from hospital after a gastro-intestinal haemorrhage arranges for subsequent reviews until the haemoglobin reaches a level of $11.6 \mathrm{~g}(80 \%)$ and until three consecutive specimens of faeces yield negative results for occult blood examination. Gastric ulcers (seven) are not differentiated and are included in the 'ulcer' group while the 'non-ulcer' group refers to the remainder.

The total of consecutive adult patients interviewed was 139; eighty-seven were males, of whom sixty-five were ulcers and twenty-two non-ulcers; and fifty-two were females, the respective numbers being twenty-five and twenty-seven. The groups were comparable in age-distribution.

\section{Results}

\section{Severity of the bleed}

The incidence of the very severe bleeds has been reduced by virtue of the exclusion of patients who had 
been submitted to emergency surgery. To fifty-one ulcer patients 133 pints of blood were administered and 89 pints to thirty-one non-ulcer patients; the average blood requirement lies between 2.5 and 3 pints/patient and there is little difference in the two groups. An arbitrary division was taken at 55 years of age; the average transfusion requirement of the older age-group differed but little from the younger in either the ulcer or non-ulcer subjects of our series.

\section{Symptomatology in relation to the two groups}

The broad gradings of 'none', 'occasional', 'intermittent' and 'more or less continuous' proved practical in defining pain and dyspeptic symptoms. Our observations, as would have been anticipated, have yielded a marked preponderance of freedom and minor discomfort in the non-ulcer group prior to the bleed but persistence of severe pain and dyspepsia was equally distributed in some $6 \%$ of the two sets of patients in the post-bleed years.

\section{Recurrent bleeds}

Thirty-two of the 139 patients had multiple haemorrhages with an aggregate of seventy-two bleeds. The incidence in the ulcer (twenty) and nonulcer (twelve) groups was in direct proportion to the original ratios as were the transfusional requirements. There was no detectable pattern of time-interval between the bleeds. No correlation was found with persisting dyspepsia or with variation in aspirin intake; no tendency to involve the later age-group and no subsequent anaemia or attributable deterioration in general health were evident. No death occurred as a consequence of a recurrent haemorrhage. Radiological re-examination of the recurrent bleeders now demonstrated a duodenal ulcer in two of the twelve formerly radiologically-negative patients.

\section{Salicylate habits}

The proportion of patients with a history of salicylate consumption prior to the haemorrhage in the ulcer group was $1: 5.3$ and in the non-ulcer group $1: 2 \cdot 8$. The average requirement of blood in the transfused subjects with a history of prior salicylate ingestion was almost equal in both groups and approximated to that of the patients with no such history.

\section{Present haematological state}

Following correction of the post-haemorrhagic anaemia no subsequent review was arranged unless the patient was referred back by the practitioner or had bled again. None of the patients had continued with iron medication for more than 3 months after the time of haematological correction. Values below $80 \%(11.6 \mathrm{~g})$ were obtained in only two men and six women. Of these eight, two were in the $70 \%$ range. Two of the women were still menstruating and the diet was distinctly suspect in three others.

\section{Liver function tests}

An abnormal value for serum albumin was accepted as below $3 \mathrm{~g} / 100 \mathrm{ml}$ since such a figure is considered to indicate liver damage in the absence of any other obvious cause for hypoproteinaemia (Baron, 1957). Values of over 4 units and $0.8 \mathrm{mg} / 100$ $\mathrm{ml}$ for the thymol turbidity and serum bilirubin levels, respectively, were judged within the abnormal range; the upper limit of the alkaline phosphatase was accepted as $13 \mathrm{King}$-Armstrong units and of the zinc sulphate turbidity as 8 units (Eastham, 1963). None of our subjects had been jaundiced. Irrespective of whether blood had been transfused the tests were conducted on all the members of the follow-up group, so that the non-transfused might offer a basis for comparison of the results. In the event this proved to be superfluous. All the results were well within the normal range with one exception (an elevated zinc sulphate turbidity). Physical examination revealed no signs of hepatic disorder in any.

\section{Blood-group distributions}

A series of 200 consecutive transfused cases was reviewed. Of the 124 ulcers the blood-group distribution was: $A$, thirty-four; $\mathrm{B}$, eleven; $\mathrm{AB}$, one; $\mathrm{O}$, seventy-six. The distribution in the non-ulcers was A, twenty; B, eight; $A B$, none; $O$, fifty. In the thirtytwo recurrent bleeders the grouping of ulcers (twenty) was: $\mathrm{A}$, five; $\mathrm{B}$, none; $\mathrm{AB}$, none; $\mathrm{O}$, fifteen; and of non-ulcers (twelve), $\mathrm{A}$, three; $\mathrm{B}$, one; $\mathrm{AB}$, none; $\mathrm{O}$, eight.

\section{Possible long-term ill-effects of the haemorrhage}

The assessment of possible ill-effects of the bleed extending into the subsequent years was made on an appraisal of the general condition of the individual in relation to his age and to any co-existing disorder. We could detect no evidence that any responsibility could be placed on the bleed for any present illhealth. This view coincided with the opinion of the individual, expressed to direct questioning on this score. A very striking feature was the lack of introspection relevant to the alimentary tract and a freedom from fear of possible future haemorrhagic recurrence.

Enquiry regarding a family history of gastrointestinal haemorrhage proved fruitless. We perceived no significant difference in the smoking or alcohol habits of the ulcer and non-ulcer groups.

\section{Discussion}

The separation of our series into ulcer and nonulcer groups must take cognisance of observer error, 
a phenomenon probably universal in all branches of medicine (Garland, 1960). An X-ray-negative group may be composed of a somewhat heterogeneous collection of pathologies but the degrees of difference in our patients will have been considerably reduced by the lapse of at least 5 years from the original bleed. It has been suggested that radiological investigation plays only a confirmatory role in the diagnosis of lesions of the stomach and duodenum and that the radiologist will make many mistakes in deciding upon the existence of a peptic ulcer (Lancet, 1960). On the other hand the accuracy of the X-ray examination has been upheld by Rawson (1965) who estimated a correct conclusion in $94 \%$; these figures were based on a retrospective study of 200 consecutive patients who were submitted to gastrectomy.

Our series demonstrates no significant difference in the severity of the bleed in ulcer and non-ulcer patients who survived the initial haemorrhagic episode without recourse to surgery and this was found also to apply in the review of those who had sustained a recurrent haemorrhage.

The natural history of peptic ulcer in general practice discerned by Fry (1964) was that of an increasing disability for between 5 and 10 years and once a 'peak' was reached a steady and progressive improvement occurred, the course being uninfluenced by medical treatment. The exclusion of those patients who have undergone gastrectomy as an elective procedure during the 5 -year period by removing a larger proportion of cases with severe symptoms may influence our figures of subsequent severe pain and dyspepsia but their infrequency corresponds to the findings of a survey of gastric and duodenal ulcers in a hospital group which revealed a behaviour similar to that of the study from general practice (Krag, 1966).

The existence of occult faecal blood loss in the majority who consume salicylates appears to be established but is probably distinct from the entity of salicylate haematemesis. As judged by the immunity from overt haemorrhage of those who continue to take the preparation subsequently and the long duration of aspirin ingestion which precedes the bleed in some, a reasonable assumption may be that salicylates are but one of the group of noxious influences whose ability to produce the haemorrhage is to a considerable degree dependent on a concomitant favourable predisposing local state however that may be induced. Jennings (1965) emphasizes the participation of tiring journeys, rushed meals and anxiety and hostility in the production of hyperaemia, hypersecretion and hyper-motility of the stomach; such situations might render the individual unduly susceptible. During the winter of the epidemic of Asian influenza, when the public was being continuously exhorted by the various media about the beneficial and preventive properties of aspirin-containing products, no increase of hospital admissions of acute upper gastro-intestinal haemorrhage ensued (Shafar \& Forgie, 1958).

Examination of the current haemoglobin levels has yielded a low value in only the occasional patient, and this applies to the multiple bleeders. The absence of anaemia is striking and suggests that anaemia will prove an infrequent event in those who have suffered an acute bleed provided the blood picture has been restored to normal, that the subsequent diet has been adequate and that no significant intercurrent disease process has evolved. In our series the acute haemorrhage has presented as a circumscribed incident which is not succeeded thereafter by any tendency to persistent or intermittent latent blood loss of any significant degree. The experience of Witts (personal communication 1966) is in agreement and he states that hypochromic anaemia is an uncommon event following the acute haemorrhage if it has been haematologically corrected. This behaviour contrasts with the predisposition for iron deficency anaemia in general to recur when treatment is stopped.

Icteric post-transfusion hepatitis presents a considerably greater problem in America than in this country. Prophylactic use of $\gamma$-globulin reduced the estimated incidence of icteric hepatitis by $75 \%$ (Mirick, Ward \& McCollum, 1965), but did not prevent hepatic involvement, the icteric merely being transformed to the non-icteric form. The asymptomatic nature of the anicteric group allows it to escape detection unless routine tests of hepatic function are instituted. It is, therefore, necessary to examine the possibility of long-term ill-effects of a condition which is clinically silent in the acute phase. No physical findings indicative of hepatic involvement were detected in the subsequent examination. The tests we employed for assessment of liver function were all found to lie within the accepted normal ranges, with but one exception. The omission of enzyme studies may be criticized (Zuckerman, 1966) but Percic et al. (1966) concluded that serum transaminase estimations are of little value in chronic liver disease and do not indicate the existing functional state but are related to the progression or inactivity of the hepatic disturbance. Walsh (1967) states that the thymol turbidity and zinc sulphate turbidity serum levels are often the last of the liver function tests to return to normal.

An association between blood group $\mathrm{O}$ and duodenal ulcer is recognized. The conclusion from a recent extensive study from Athens (Merikas, Christakopoulos \& Petropoulos, 1966) is that this association may in whole or in part be explained by the strong relationship between group $\mathbf{O}$ and bleeding. Langman \& Doll (1965) also conclude that 
group $O$ patients are more likely to suffer from bleeding. Our own figures are small but the high incidence of group $\mathbf{O}$ in the non-ulcer group suggests that a useful future investigation may be the examination of a larger series to determine whether the preponderance of this blood group is a concomitant of non-ulcer haemorrhage as well as ulcer haemorrhage.

Our findings reveal a sufficiently close parallelism of behaviour between our select group of ulcer patients and the non-ulcer group as to invite consideration of the possibility of the existence in both of closely related local factors concerned in the genesis of the bleed. The presence of a peptic ulcer does not necessarily imply that it is the site of the haemorrhage which may for example arise from a co-existent acute gastric erosion (Bogoch, 1963). Initially radiologically negative cases may at some later date display a duodenal ulcer (Jones, Read \& Stubbe, 1959) but the assumption is not definite that the ulcer was present on the occasion of the haemorrhage. The practice of Forty $(1964,1965)$ of submitting the gastrectomy specimen routinely for histological examination has led him to conclude that the changes of chronic gastritis are consistently present in gastric and duodenal ulcer and he states that the bleeding arises from microscopic erosions as often as it does from an ulcer; he considers that failure to appreciate this aspect is a consequence of radiography having focused undue attention on the ulcer itself. That the incidence of ulcer is so high in our 139 cases may be referable to regurgitation of duodenal contents through a pylorus rendered incompetent by a deforming duodenal ulcer; duodenal reflux produces chronic gastritis (Capper, Airth \& Kilby, 1966). The precipitation of a disordered state of the microvasculature of the stomach merits consideration as a possible underlying mechanism for the origin of the bleed in some. Such has been demonstrated to occur in neurogenic haematemesis by Doig \& Shafar (1956) who emphasize the decisive role of the superficial mucosal haemorrhage. Katz, Siegel \& Sussman (1966) have reported haemorrhagic changes in the neck area and lamina propria of the gastric glands in the gastric biopsy specimens of patients with haematemesis even when no obvious abnormality was visible on gastroscopic examination. The conclusions of Djorup (1965) are in accord; he found that both in chronic and acute ulcers, in twothirds of the cases the haemorrhage arises in damaged veins and capillaries and that the subepithelial haemorrhage is a decisive factor in its pathogenesis. The cut edge of the stomach bleeds freely during the operation for the arrest of haemorrhage from an acute ulcer (Banning et al., 1965) and also pertinent is that a patient operated upon for repeated haemorrhages from a peptic ulcer-even as an interval procedure-is more likely to have excessive oozing of blood into the stomach than is found when the procedure has been adopted because of pain, perforation or obstruction (Lancet, 1965). Langman (1965), investigating the relationship between pre-operative bleeding and bleeding occurring some time after gastrectomy undertaken for duodenal ulcer, reported a much greater frequency of such an association than in a control series of successive operations for duodenal ulcer. Palmer (1961) in a study of sixty-six ulcer patients who were submitted to a further surgical procedure, specifically on account of recurrent haemorrhage, discovered a stomal ulcer in only thirty-three cases; in fourteen the basis was erosive gastritis and in five erosive oesophagitis. A follow-up study of 351 patients on whom subtotal gastrectomy had been performed for peptic ulcer and who had survived at least 10 years from the date of the operation was conducted by Kiefer (1959); forty-eight had bled at least once subsequently but an ulcer crater was demonstrable in only seventeen. Of 126 ulcer patients who had had a haematemesis fifty-one $(40 \%)$ bled subsequently and, moreover, subtotal gastrectomy was associated with a significant failure to prevent subsequent bleeds; Serebro \& Mendeloff (1966) consider that the peptic ulcer patient is as likely to bleed from eroded mucosa as from the specific ulcer itself. This tendency to recurrence of bleeding following gastrectomy is not peculiar to the ulcer case alone. Thus, Appleman \& Beahrs (1965) record that of sixteen patients in whom gastrectomy was performed for unexplained gastro-intestinal bleeding, five bled at a later date.

When gastroscopy revealed an acute erosive gastritis as the bleeding source, Katz et al. (1966) obtained confirmation of an abnormal histological state in $87.5 \%$ by suction biopsy; very significantly, however, a similar high frequency $(79 \%)$ of positive histological findings was obtained in undiagnosed cases of bleeding, which suggested that bleeding erosive lesions may exist undetected by gastroscopy. In another study by Katz et al. (1965) in which gastric biopsy material was examined routinely in twenty-four consecutive admissions for haematemesis other than that attributable to bleeding oesophageal varices, no single type of chronic gastritis was evident, nine revealing no signs of such pathology while ten manifested early atrophic gastritis and four atrophic gastritis; the essential lesions, in different degrees, were denudation of superficial epithelium, haemorrhage into the neck of the glands and haemorrhage in the lamina propria of the glands. Djorup (1965) also emphasized that sub-epithelial haemorrhage appears to play a decisive role in the pathogenesis but that as yet its causal mechanism remains unknown. The vascular changes 
are reminiscent of those which ensue in the gastric mucosal vessels in the rat in response to restraint stress (Guth \& Hall, 1966) as well as those which were described by Doig \& Shafar (1956) in neurogenic haematemesis. We are of the opinion that our results coupled with such data from the literature as we have outlined lend support to the tentative hypothesis of a common process of vascular disturbance whose localization may vary from the oesophagus to the duodenum, as the precipitating lesion of the acute bleed in some peptic ulcer and non-ulcer patients.

\section{Acknowledgments}

We are grateful to the Manchester Regional Hospital Board for a grant towards the expenses of the study and to Mr J. Lowe, B.SC., for his considerable assistance.

\section{References}

Appleman, R.H. \& Beahrs, O.H. (1965) Surgical management of gastrointestinal hemorrhage from an undetermined source. Proc. Mayo Clin. 40, 121.

Banning, A., Baron, A., Kopelman, H., Lam, K.L. \& WARREN, P. (1965) Bleeding peptic ulcer. Brit. med. J. 2, 781.

Baron, D.N. (1957) Essentials of Chemical Pathology. Cambridge University Press.

BoGOCH, A. (1963) Gastroenterology (Ed. by H. L. Bockus) 2nd edn, Vol. 1. Saunders, Philadelphia.

CAPPER, W.M., AIRTH, G.R. \& KilbY, J.O. (1966) A test for pyloric regurgitation. Lancet, ii, 621.

DJORUP, F. (1965) The source of bleeding in acute gastric hemorrhage. Acta Chir. Scand. Suppl., 343.

Dolg, A. \& Shafar, J. (1956) Gastric haemorrhage in acute intracranial vascular accidents. Quart. J. Med. 97, .

Eastham, R.D. (1963) Biochemical Values in Clinical Medicine. John Wright, Bristol.

FORTY, F. (1964) Correspondence. Gastrectomy. Lancet, ii, 416.

FORTY, F. (1965) Correspondence. Bleeding, before and after. Lancet, ii, 136.

FRY, J. (1964) Peptic ulcer: a profile. Brit. med. J. 2, 809.

GaRLAND, L.H. (1960) The problem of observer error. Bull. N.Y.Acad. Med. 36, 570.

Guth, P.H. \& Hall, P. (1966) Microcirculatory and mast cell changes in restraint-induced gastric ulcer. Gastroenterology, 50, 562.
JENNINGS, G.H. (1965) Causal influences in haematemesis and melaena. Gut, 6, 1.

Jones, F. Avery, Read, A.E. \& Stubbe, J.L. (1959) Alimentary bleeding of obscure origin. Brit. med. J. 1, 1139.

Katz, D., Siegel, H., Paulo, D. \& Sussman, H.M. (1965) Gastric biopsy in patients with acute upper gastrointestinal hemorrhage. Gastrointest. Endoscopy, 12, 36.

Katz, D., Siegel, H. \& Sussman, H. (1966) Gastric biopsy in acute upper gastrointestinal hemorrhage and controls: histological confirmation of erosive gastritis seen gastroscopically. Gastroenterology. 50, 852.

KiEfER, E.D. (1959) Life with a subtotal gastrectomy. A follow-up study ten or more years after operation. Gastroenterology. 37, 434.

KRAG, E. (1966) Gastric acid secretion related to prognosis in peptic ulcer. A long-term follow-up study. Acta med. scand. 180, 461.

Lancet (1960) Radiographic detection of primary peptic ulcer. i, 639.

Lancet (1965) Bleeding before and after. ii, 73.

LANGMAN, M.J.S. (1965) Relationship between pre-operative bleeding and perforation and bleeding after operations for duodenal ulcer. Gut, 6, 134.

Langman, M.J.S. \& Doll, R. (1965) ABO blood group and secretor status in relation to clinical characteristics of peptic ulcers. Gut, 6, 270.

Merikas, G., Christakopoulos, P. \& Petropoulos, E. (1966) Distribution of ABO blood groups in patients with ulcer disease. Its relationship to gastroduodenal bleeding. Amer. J. dig. Dis. 11, 790.

MiricK, G.S., WaRD, R. \& McCollum, R.W. (1965) Modification of post-transfusion hepatitis by gamma globulin. New Engl. J. Med. 273, 59.

PALMER, E.D. (1965) Diagnosis of Upper Gastrointestinal Haemorrhage. Thomas, Springfield, Illinois.

Percic, V., Stojsic, S., Hegedis, T. \& Ostrogonac, L. (1966) Serum transaminase activity in liver and biliary tract diseases. Amer. J. Gastroent. 45, 348.

Rawson, M.D. (1965) Accuracy in diagnosis of gastric and duodenal lesions by general practitioners, hospital clinicians and radiologists. Lancet, i, 698.

Shafar, J. \& Forgie, R.E. (1958) Correspondence. Aspirin and peptic ulcer. Lancet, ii, 373.

Serebro, H.A. \& Mendeloff, A.I. (1966) Late results of medical and surgical treatment of bleeding peptic ulcer. Lancet, ii, 505.

WALSH, M.P. (1967) Biochemical investigation of liver disease. Hosp. Med. 1, 487.

ZuCKERMAN, A.J. (1966) Blood transfusion and infective hepatitis. Brit. med. J. 2, 1136. 